NEWS AND VIEWS

\section{Inheritance of Old Age}

THE most persuasive explanation so far for the molecular basis of ageing receives strong support from the experiments now carried out by $\mathbf{R}$. Holliday (see page 1224). Some years ago, L. Orgel suggested that the crucial molecular events that lead to senescence are the occurrence and accumulation of errors in certain classes of enzymes. Orgel argued that mistakes during the synthesis of most proteins would be irrelevant to ageing because the altered molecules would simply be diluted out. But if any of the enzymes involved in DNA synthesis or protein synthesis contained errors which blunt their specificity, the result may be to induce errors in the proteins being synthesized. Once the protein synthesizing machinery of a cell is contaminated in this way, errors would accumulate exponentially, and the cell would be unable to rectify the fault, which would be passed on through the cytoplasm to daughter cells. Then, once a critical threshold was reached, the cells would suffer an "error catas. trophe" and die. A small body of evidence has accumulated since 1963, which is at least compatible with Orgel's hypothesis, and Holliday's latest series of experiments, with two species of fungi, fulfil one of its chief predictions-that an increase of the frequency of errors during protein synthesis should accelerate ageing.

Two years ago, Harrison and Holliday reported (Nature, 213,$990 ; 1967)$ that feeding Drosophila larvae with analogues of amino-acids or the antibiotic streptomycin, which might be expected to increase the frequency of mistakes during protein synthesis, shortens the lifespan of the adult flies which emerge. Clearly this result is compatible with Orgel's hypothesis although it by no means constitutes a proof. Indeed, there are several other equally plausible interpretations. For example, proteins of Drosophila are known to have long half-lives so that an altered protein made in the larva might survive in the adult, malfunction and leave the adult fly less vigorous than normal flies. This in turn might accelerate the normal but quite separate ageing mechanism.

In his latest experiments with two species of fungi, Holliday has used the same basic approach as in these experiments with Drosophila. He has found a correlation of accelerated ageing with increased frequency of mistakes in protein synthesis. One of the species he used, Podospora aserina, undergoes a very characteristic growth cycle. On a solid medium the fungal hyphae grow at a constant rate and then suddenly stop growing, swell up, become pigmented and finally burst and die. On Orgel's hypothesis, this pattern of growth can be explained by assuming that the hyphae can grow quite happily until their protein synthesizing machinery is contaminated by so many altered proteins that a threshold is reached. Thereafter, further errors result in an "error catastrophe" and the hyphae die.
Holliday has now found that by manipulating the growth conditions so as to encourage the induction of mistakes during protein synthesis, the ageing of the Podospora hyphae is accelerated.

The other species of fungus used in these studies, Neurospora crassa, permits a rather more critical test of Orgel's hypothesis. Neurospora, unlike Podospora, normally grows for very long periods without any apparent ageing but eventually enters a stop start cycle of growth known to mycologists as the "stopper" stage. At present it is not known whether the cause of this irregular growth is the same as that of senescence of Podospora, but certain mutants of Neurospora have been isolated which mimic the growth pattern of Podospora. One of these grows for a while at a constant rate and then quite suddenly stops growing and dies.

The question is whether this mutant strain, which is called the natural death or $n d$ strain, makes more errors during protein synthesis than the wild type. Holliday believes that his experiments show that it does. The experiment involves crossing the $n d$ mutant strain with another mutant strain and showing, in a sense, that two wrongs make a right, at least for a while. The second mutant strain used in the cross contains a mutation in the gene which specifies one of the enzymes involved in the metabolism of the nucleic acid base adenine. As a consequence, the strain needs to be supplied with adenine in order to grow. This mutation is, however, "leaky"-in other words the genetic defect can be rectified, in a proportion of the gene product, at the stage at which the genetic instructions are translated into protein by the cellular machinery. If the $n d$ mutation results in an increase in the number of errors during protein synthesis, it should increase the chance of rectifying the adenine enzyme mutation because it may induce mistakes which exactly reverse the adenine mutation. This is the result Holliday has obtained. Crosses between $n d$ and adenine mutants grow better than crosses between wild type and adenine mutants under conditions in which the supply of adenine is limiting. Holliday says there may be several alternative interpretations of any one of his results, but Orgel's is the simplest unifying hypothesis to explain the results obtained with Drosophila, Podospora and Neurospora. The crucial test now is to show that the cytoplasm of aged cells contains more abnormal proteins than the cytoplasm of young cells of the same species.

\section{ASTRONOMY \\ Leonids above Par \\ from our Astronomy Correspondent}

The Leonid meteor shower which occurs in November each year, but with a dramatic increase in activity 